\title{
Analysis and Research on Physical Training Test Based on Calculation Matrix and GM (1, N) Model
}

\author{
Di Liu \\ Sports Department, Northeast Forestry University, Harbin, China \\ I_iu_di@126.com
}

\begin{abstract}
Keywords: Fitness Training; Contrast test; Mathematical statistics; GM ( $1, \mathrm{~N}$ ) model; Vector matrix; Least square method; Curve fitting
\end{abstract}

\begin{abstract}
The basketball sport is an important part of China's university physical education curriculum, and it plays an important role in guiding students to healthily spread sports culture. This paper used the concept of fitness training in the basketball sports, and it had the experimental researches on the fitness training of basketball sports, at the same time it was through the methods of contrast test, mathematical statistics, mathematical modeling to have analysis on the results of fitness training. The results show that fitness training in the university basketball can get better training effect. Therefore the concept of fitness training can be applied in the reform of sports education in our country, and it can establish the new teaching mode. It is also known that the aspects of age and weight have significant impact on the performance of basketball sports' fitness training.
\end{abstract}

\section{Introduction}

The basketball sport is the important component of China's university physical education curriculum, and it is very popular with the students. At the same time the basketball sport plays an indispensable role in ensuring students' healthy body, promoting cultural exchange and training the modern talent. Along with the further development of our country's reform and opening, the teaching mode of university sports basketball course has fallen behind a lot, and it is necessary to develop the new teaching mode.

At present, the fitness is put forward as one of the main goals in the international society. Along with the increasing reform of our country's quality education, the concept of fitness is gradually integrated into the health education field in our country[1]. The events organization of the United States sports health and dance in the beginning is to help the children understand the value and significance of the sports activities, and it also can cultivate the good behavior habits. Then the continuous development makes this idea finally form the concept of fitness. The idea is slowly spread to all over the world, and it causes great repercussions in Japan, Britain, Australia, Hong Kong and other regions. This idea has good performance of guiding the children to take an active part in sports activities, forming the good life habit, improving the physical health. Then the idea is spread to the university sports, and it can reflect the new trend of sports development. The new tendency includes the lifelong sports, personalization and health.

Now in our country, many universities have carried out the fitness sports teaching activities. Physical fitness can be put forward to have a revolutionary influence on sports teaching of our country, but fitness sports teaching mode of many universities is in the groping stage. At the same time some teachers often do not have sufficient understanding of the fitness, and they think that it is mainly related to the physical exercise. So they make the student try hard to have physical exercise, and they do not pay attention to the improvement of the overall physical fitness, finally this leads to the poor teaching effect. So the development of the researches on the fitness training of the teaching mode in our country is very necessary.

\section{Simple introduction of fitness}

A. Definition of fitness

Domestic and foreign scholars have a lot of definition methods of understanding the concept of 
fitness, and the main methods have the following two kinds of interpretation ways[2]. The general fitness mainly refers to the body's adaptive capacity (adaptive capacity includes the human body's abilities of adapting to the external environment, resisting the diseases, and human body organs' ability of adjusting the change of internal environment). The special fitness mainly refers to the fitness that is related to the health and competitive activities (the fitness of competitive activities can be divided into general leisure activities and competitive games, and athletes' indispensable special sport fitness).

The concept of health fitness is that the human body has enough energy and vitality in processing of everyday activities, and the body doesn't feel tired, at the same time the human body has enough energy to enjoy leisure activities and deal with the emergency[3].The concept of general sports fitness is the autonomic regulation ability of the sensitivity, speed of movement, balanced capacity, coordinate ability, explosive force and other factors of the body when people participate in sports activities. And when people takes part in some sports activities, the special athletic ability of human body can be called as special fitness, such as the suppleness of gymnastics, balanced capacity of figure skating and so on.

B. Compositional factors of fitness

Compositional factors of fitness is as Figure 1 shown.

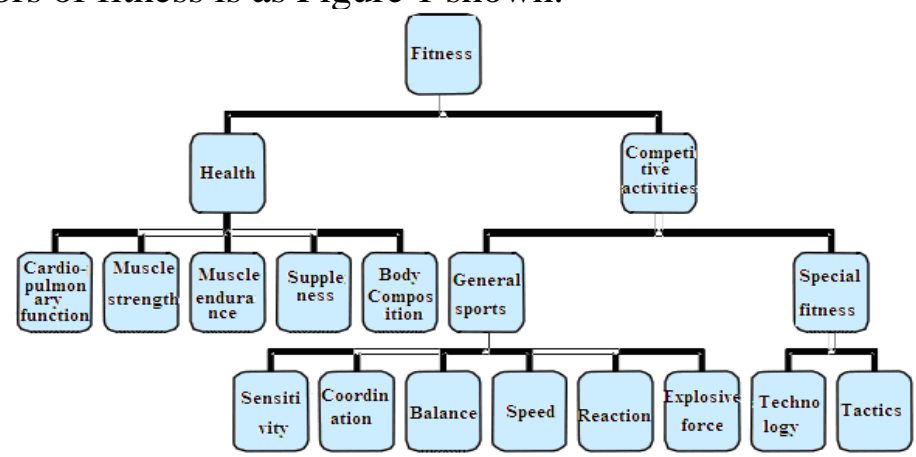

Figure 1. Compositional factors of fitness system

\section{Introduction of basketball fitness training theory}

A.Meaning of basketball fitness training

The content of basketball fitness training mainly has the following several aspects. The first aspect is to enhance the human body's cardio-pulmonary function, muscle strength, endurance, suppleness and fat content and other indexes. It is to improve health fitness of human body, and in other words it is to change the human body form and improve the body environment's adaptive capacity of basketball sports[4].The second aspect is human body's sensitivity, coordinate ability, balanced capacity, speed of movement, reaction speed, explosive force and other key elements of fitness, and they are called as adaptability of physical quality. The third aspect is the special fitness, and it is mainly expressed in the basketball skills and the improvement of actual combat.

Through the appropriate training content and skills, certain load movement, regular schedule, appropriate frequency exercise, human internal environment and body form can gradually have adaptive changes. The improvement of the level of basketball and the actual combat skills' system training is the basketball fitness training. It is radically different from the athletic training that has been used in China.

B.Principle of basketball fitness training

The human body system has the stability and adaptability. The stability refers to that when the outside temperature and humidity occur changes, people's internal temperature, body fluids and other interior environments are changing in a certain range without big changes. When the human body has external stimulation for a long time, the human body form and the internal environment will be gradually changing in order to adapt to the requirements of the external stimulation. When the human body has continuous life activities, the reason of human body's existence is the human body function's adaptability and stability of internal and external environment. People's adaptive capacity can be divided into short-term adaptation and long-term adaptation[5]. When the external environment of the human body occurs changes, the human body has changes in order to adapt to the short time needs of the environment, and when the body leaves the environment and goes back to the 
original environment, then the human body's function recovers the original state, this situation is called the temporarily adapt. When the human body has periodic stimulation for a long time, it can make human body form and function have substantial changes, and when there is no stimulation, the changes are not going to disappear after a long time, this change can be called the long-term adaptation.

So we can see that basketball fitness training is to achieve the purpose of making human body adapt to the basketball. It is through the long-term, regular training that has certain load, when students' basketball capabilities have improvement, it can be found that students' cyclic system, muscle strength, breathing rate, neural response and a series of biological characteristics are getting better and better[6].

\section{Experimental research of basketball teaching}

\section{A.Test purpose}

This paper used comparative teaching tests to have researches on the teaching mode of fitness training in the university basketball courses, the content of the researches also referred to that whether the fitness training can improve the students' basketball ability, and it established the model of the influence of fitness training's each factor on the improvement of human performance.

\section{B.Test methods}

The five main indexes that include the endurance, cardio-pulmonary function, suppleness, muscle strength and body form constitute the fitness. At the same time, it also can be simply divided into muscle fitness (it includes muscle strength and muscular endurance), body shape (it is mainly displayed in the body height, weight and other aspects)[7]. But the most important things are the cardio-pulmonary function and body suppleness, because they can represent the performance of human body's internal environment system. According to the characteristics of basketball sports, this test was mainly based on the different ages, heights and weights to test the cardio-pulmonary function.

\section{C.Test object}

The junior students in a university were selected as the research object (this mainly considered that the investigations of the junior students' sports curriculum were over, and they didn't have sports, this situation had little influence on the test)[8]. The test randomly selected 56 students to take part in the basketball special training course, and they didn't participate in any other sports activities. Test object is divided into two groups, and it respectively carried on t test of their four fitness indexes. Then it had data statistics analysis on the four indicators' testing results to avoid students' abnormal psychology, and it counted the results that didn't reach the condition of $\mathrm{P}>0.05$. The results are shown in Table 1.

\begin{tabular}{|c|c|c|c|}
\hline TABLE I. & ISTICAL & ALYSIS OH & TT OBJECT \\
\hline Groups & Age & Height /mm & Weight /kg \\
\hline Experimental group & $21.2 \pm 0.54$ & $176.54 \pm 5.41$ & $67.18 \pm 6.45$ \\
\hline Comparative group & $19.99 \pm 1.56$ & $175.75 \pm 4.45$ & $68.52 \pm 6.46$ \\
\hline $\mathrm{t}$ & 1.831 & 0.101 & 0.595 \\
\hline $\mathrm{P}$ & $>0.05$ & $>0.05$ & $>0.05$ \\
\hline
\end{tabular}

\section{D.Test results}

The statistics of test results are shown in Table 2, from the table we can see that when the students have basketball fitness training, the four test indexes of the experimental group and the comparative group have $t$ test. The four indicators of cardiac function, muscle strength, suppleness and fat content have reached significant difference of statistical significance $(P>0.05)$. Results show that development of fitness training can efficiently improve the level of basketball training in China. 
TABLE II. COMPARISON OF EXPERIMENTAL GROUP’S FITNESS AND COMPARATIVE GROUP'S FITNESS AFTER TEST

\begin{tabular}{|c|l|l|l|l|}
\hline & \multicolumn{1}{|c|}{ Step test } & \multicolumn{1}{|c|}{$\begin{array}{c}\text { Muscle strength } \\
\text { /kg }\end{array}$} & \multicolumn{1}{c|}{$\begin{array}{c}\text { Suppleness } \\
\text { /cm }\end{array}$} & \multicolumn{1}{c|}{$\begin{array}{c}\text { Fat content } \\
/ \%\end{array}$} \\
\cline { 2 - 5 } & $\mathrm{M} \pm \mathrm{SDtp}$ & $\mathrm{M} \pm \mathrm{SDtp}$ & $\mathrm{M} \pm \mathrm{SD}$ tp & $\mathrm{M} \pm \mathrm{SDtp}$ \\
\hline $\begin{array}{c}\text { Experimental } \\
\text { group }\end{array}$ & $46.14 \pm 4.56$ & $51.45 \pm 7.21$ & $14.21 \pm 5.41$ & $17.89 \pm 2.98$ \\
\cline { 2 - 5 } & $0.504>0.05$ & $0.201>0.05$ & $0.104>0.05$ & $0.478>0.05$ \\
\hline $\begin{array}{c}\text { Comparative } \\
\text { group }\end{array}$ & $44.24 \pm 5.71$ & $50.47 \pm 5.67$ & $14.74 \pm 3.56$ & $17.27 \pm 3.54$ \\
\hline
\end{tabular}

\section{GM (1, N) model of basketball fitness training}

\section{A.GM $(1, \mathrm{~N})$ model}

There has $\mathrm{n}$ variables of $x_{1}, x_{2}, \ldots . . x_{n}$ and the $N$ sequences. So the original data matrix is[9]:

$$
X^{(0)}=\left[\begin{array}{cccc}
x_{1}^{(0)}(1) & x_{1}^{(0)}(2) & \cdots & x_{1}^{(0)}(m) \\
x_{2}^{(0)}(1) & x_{2}^{(0)}(2) & \cdots & x_{2}^{(0)}(m) \\
\vdots & \vdots & \ddots & \vdots \\
x_{N}^{(0)}(1) & x_{N}^{(0)}(2) & \cdots & x_{N}^{(0)}(m)
\end{array}\right]
$$

Because the initial data has error and some random disturbance in the monitoring process, the original data should have smoothing and accumulated generating operation, then it can get the accumulating generator matrix[10]:

$$
X^{(1)}=\left[\begin{array}{cccc}
\sum_{i=1}^{2} x_{1}^{(0)}(i) & \sum_{i=1}^{3} x_{1}^{(0)}(i) & \cdots & \sum_{i=1}^{m} x_{1}^{(0)}(i) \\
\sum_{i=1}^{2} x_{2}^{(0)}(i) & \sum_{i=1}^{3} x_{2}^{(0)}(i) & \cdots & \sum_{i=1}^{m} x_{2}^{(0)}(i) \\
\vdots & \vdots & \ddots & \vdots \\
\sum_{i=1}^{2} x_{N}^{(0)}(i) & \sum_{i=1}^{3} x_{N}^{(0)}(i) & \cdots & \sum_{i=1}^{m} x_{N}^{(0)}(i)
\end{array}\right]
$$

It establishes the matrix[11]:

$$
B=\left[\begin{array}{cccc}
-\frac{1}{2}\left(x_{1}^{(1)}(2)+x_{1}^{(1)}(1)\right) & x_{2}^{(1)}(2) & \cdots & x_{N}^{(1)}(2) \\
-\frac{1}{2}\left(x_{1}^{(1)}(3)+x_{1}^{(1)}(2)\right) & x_{2}^{(1)}(3) & \cdots & x_{N}^{(1)}(3) \\
\vdots & \vdots & \ddots & \vdots \\
-\frac{1}{2}\left(x_{1}^{(1)}(m)+x_{1}^{(1)}(m-1)\right) & x_{2}^{(1)}(m) & \cdots & x_{N}^{(1)}(m)
\end{array}\right]
$$

It establishes the constant vector matrix[12]:

$$
Y_{N}=\left[\begin{array}{llll}
x_{1}^{(0)}(2) & x_{1}^{(0)}(3) & \cdots & x_{1}^{(0)}(m)
\end{array}\right]^{T}
$$

It uses the least squares method to obtain the coefficient matrix[13]:

$$
\hat{a}=\left[\begin{array}{c}
a \\
b_{1} \\
b 2 \\
\vdots \\
b_{M-1}
\end{array}\right]=\left[\begin{array}{ll}
B^{T} & B
\end{array}\right]^{-1} B Y_{N}
$$

It establishes the differential equation[14]:

$$
\frac{d x_{1}^{(1)}}{d t}+a x_{1}^{(1)}=b_{1} x_{2}^{(1)}+b_{2} x_{3}^{(1)}+\cdots b_{N-1} x_{N}^{(1)}
$$

It calculates the differential equation to get incident response function, and then it gets the GM (1, N) mathematical model[15]: 


$$
\hat{x}_{1}^{(1)}(t+1)=\left[x_{1}^{0}(1)-\sum_{i=1}^{N} \frac{b_{i-1}}{a} x_{i}^{(1)}(t+1)\right] e^{-a t}+\sum_{i=2}^{N} \frac{b_{i-1}}{a} x_{i}^{(1)}(t+1)
$$

B.The selection of variables

This test involved three influence factors, and they were age, height and weight. The three aspects were the variables, in order to have convenient calculation in GM $(1, N)$ model, they could be respectively replaced by $X_{1} 、 X_{2} 、 X_{3}$.

C.Analysis of model's results

Through the above results of GM $(1, \mathrm{~N})$ mathematical model, we can get the proportions of all kinds of influence factors in the basketball fitness training, and they are shown in Table 3. From the data in the table we can see that the age, height and weight have great influence on the basketball fitness training.

TABLE III. THE PROPORTIONS OF GM $(1, \mathrm{~N})$ MODEL'S INFLUENCE FACTORS IN THE BASKETBALL FITNESS TRAINING

\begin{tabular}{|c|l|l|l|l|}
\hline & \multicolumn{1}{|c|}{ Step test } & $\begin{array}{c}\text { Muscle } \\
\text { strength }\end{array}$ & \multicolumn{1}{|c|}{ Suppleness } & $\begin{array}{c}\text { Fat } \\
\text { content }\end{array}$ \\
\hline$X_{\text {Age }} X_{1 \%}$ & 44.3 & 53.1 & 45.3 & 69.5 \\
\hline $\begin{array}{c}\text { Height } X_{2 / \%} \\
\text { Weight } X_{3 / \%}\end{array}$ & 35.4 & 36.4 & 12.4 & 21.6 \\
\hline
\end{tabular}

\section{Conclusion}

This paper introduced the popular concept of fitness in the international society, and the fitness is applied to the basketball training in the universities. This paper explored the mechanism of basketball sports fitness training, and it paid more attention to the experimental research of university basketball fitness training, and it used the mathematical models to have analysis on the various influence factors' proportions of the basketball fitness training. It got the following conclusions:

(1) At present university basketball teaching of our country has fuzzy concept of fitness, and they don't have scientific teaching method. This situation can lead to difficult reform of basketball education mode in our country.

(2) Through the experimental researches we can see that the fitness training of basketball sports is the relatively effective training method, and it should be popularized in the universities of our country.

(3) Through analysis of mathematical modeling, the results show that age and weight have great influence on the basketball fitness training.

\section{References}

[1] Sewall L, Michelli.Strength Training for Children.J Pediatr Orthop, 2009(06):36-39.

[2] Saltin B, Gollnick PD.Skeletal Muscle Adaptability. Significance for Metabolism and performance Handbook of Physiology ,American Physiology Society, 2010(03):94-97.

[3] American Alliance for Health.Physical complete Recreation and Dance. Physical Education for Lifelong Fitness ,United States: Human kinetics, 2009(02):17-20.

[4] Xianpeng Lin. Foreign sports dynamic.State physical culture and sports information institute, 2011(10):53-56.

[5] Wenxuan Yang. Sports principle.Cambridge: Beijing sports university press, 2010:75-78.

[6] Hansheng Huang. Sports education training theory and method.Higher education press, 2011:56-61.

[7] Zhengwei Gong, Yanling Li. Try to discuss the sustainable development of the sports departments in normal universities of our country.Journal of sports science, 2010(06):24-26.

[8] Baocheng Wang. Basic theory and content of basketball players' physical ability training.Journal of capital institute of physical education, 2011(11):41-43.

[9] Hua Yang. The nature, characteristics and law of basketball sports.Journal of Chengdu sports college, 2010(07):59- 62.

[10] National sports colleges and universities teaching material committee. Sports physiology ,People's sports press, 2010:27-30.

[11] Maijiu Tian, Qun Xiang. Training theory ,Beijing: people's sports press, 2011:71-73.

[12] Chunbao Ma. Discussion of basketball movement's aerobic and anaerobic metabolism ability from perspective of exercise biochemistry, sports biochemistry in the application of sports 
practice .Higher education press, 2011:49-51.

[13] National physical culture institute teaching committee basketball teaching material group. Basketball advanced tutorial.People's sports press, 2010:67-70.

[14] Shian Wang. Basketball.Beijing sports university press, 2009(11):15-18.

[15] The ministry of education. Physical education and health course standard.Beijing normal university press, 2011(07):63-65. 